\title{
A Decision-making Module for Fertilization and Irrigation Control System in Rice Farming Using Markov Chain Process and SARSA Algorithms
}

\author{
Peace Bamurigire $^{1+}$, Anthony Vodacek ${ }^{2}$, Kayalvizhi Jayavel $^{3}$ and Said Rutabayiro Ngoga ${ }^{4}$ \\ ${ }^{1}$ African Center of Excellence in Internet of Things, College of Science and Technology, University of \\ Rwanda, Kigali, Rwanda \\ ${ }^{2}$ Chester F. Carlson Center for Imaging Science, Rochester Institute of Technology, Rochester, NY, USA \\ ${ }^{3}$ School of Computing, SRM Institute of Science and Technology, Kattankulathur, Tamil Nadu, India \\ ${ }^{4}$ Rwanda Information Society Authority, Kigali, Rwanda
}

\begin{abstract}
The merging of modern sensor technologies, the Internet, and advanced control of irrigation and fertilization with an Internet of Things (IoT) approach allow a relatively precise control of agriculture. This IoT approach can thereby increase the resilience of agricultural systems in the face of complex demands for water and fertilizer use, even in countries such as Rwanda with low levels of economic development as long as appropriate and low cost technologies are used. In this work, we add to our previous IoT design for an irrigation system by adding a fertilization system. The proposed low cost system will provide individual farmers fertilization and irrigation options informed by reservoir capacity, water level, predicted rainfall, and temperature along with soil condition and $\mathrm{pH}$. IoT data are assessed in the context of rice growth stages as a Markov Chain process, or in the case of IoT system fault, assessed using the SARSA temporal difference technique. Simulations for the Muvumba Valley rice project in Northeast Rwanda demonstrate the potential of this IoT system to increase rice yield while decreasing fertilizer and water use.
\end{abstract}

Keywords: IoT, Irrigation, Fertilization, Rice, Smart Farming, Sensors, Mobile Phone, Cloud Storage, Wireless.

\section{Introduction}

In previous research, we demonstrated a model to control an Internet of Things (IoT) system for rice irrigation using approaches that are appropriate for Rwanda, a country with low economic development [1]. Here, we expand this IoT system to include models for controlling fertilization and irrigation. Rice is the second most important cereal and staple food in Rwanda but the demand is largely met by importing rice from different countries around the world. The government thus supports irrigation schemes to increase agricultural resiliency and self-sufficiency in areas such as Muvumba Valley that are otherwise too dry for optimal rice growth. With irrigation and fertilization Muvumba Valley can support two rice crops per year due to the two rainy seasons in this equatorial region; Season A is March to August and Season B is September to January. According to [2], the current irrigation and fertilization system is manual, where farmers irrigate and apply fertilizer according to instruction of the local agronomist. Conflicts arise in this system because there has to be a collective request of the farmers to achieve maximum water consumption and water control structures are minimal. Fertilizer application is made without regard to soil condition due to lack of adequate and timely soil testing [3]. The objective of this work is to demonstrate through simulation a system, which uses IOT to control both irrigation and fertilization with fault tolerance in order to increase rice yield.

\section{Methods}

Our IoT system is designed for the Muvumba Valley Rice Plantation located in Nyagatare District, Eastern Province, Rwanda (1.302 S, 30.310 E). This growers' cooperative has a total area of 1,750 ha

\footnotetext{
+ Corresponding author.

E-mail address: bgpeace20@gmail.com
} 
distributed among 1,085 farmers. This system is an extension of the irrigation control system described in [1]. The flowchart of the IoT system (Figure 1) has two pathways, one is the fully functioning automatic system (white) and the second only initiates if there is a fault in the automatic system (gray). The fault pathway provides actionable recommendations for the farmer until the fault is fixed. Farmer receive system status and actions via smart phone or SMS whether the system is fully operational or in a fault state. With indication of a fault, the farmer is notified of the fault and irrigation and fertilizer recommendations are communicated, but the farmer must manually control the system. Different modelling processes are used to control irrigation and fertilization for the data rich normal operation and the data starved pathway when a fault occurs. In the automatic pathway, the irrigation (WL) and fertilization decision is modeled as a Markov chain process (MCP) [4]. The IoT system will check the water level (WL) and fertilizer level (FL) in the field and assess predicted rainfall and temperature by Web service. Irrigation is handled as in [1]. If fertilizer (NPK/Urea) is needed the system will engage to add and deliver the appropriate amount of fertilizer through the irrigation system. This IoT system will allow individual farmers to control the irrigation and fertilization of their individual plots to overcome this limitation of the current practice. When there is a fault in the IoT system the pathway switches to the SARSA $(\lambda)$ temporal difference method to propagate the recommended control to the farmer for manual control until the IoT system is repaired [5][6].

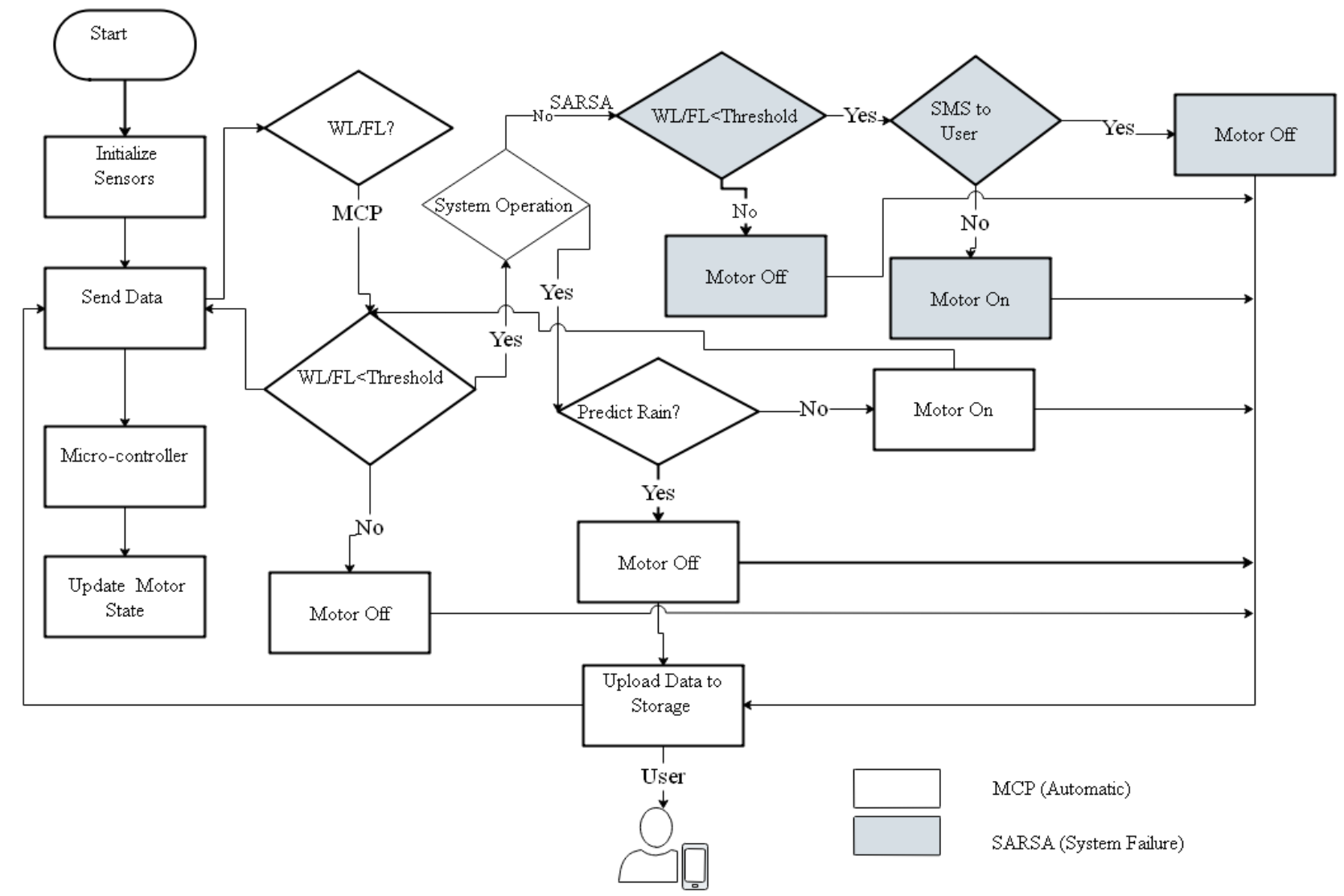

Fig. 1: Flowchart of the irrigation and fertilization optimization system. White boxes are the MCP modeling flow and the gray boxes are the SARSA modeling flow.

MCP has been demonstrated to be a valid modeling approach for agricultural crop systems [4]. Water and fertilizer requirements for rice change for each stage of growth so the MCP deals with this set of possible stages $\mathrm{S}$ and the set of possible actions $\mathrm{A}$, where the effects of an action taken in a stage depend only on that stage and not on the prior history. As in [1] we use MCP to model the probabilistic transitions among a set of stages $S=\left\{s_{1}, s_{2}, \cdots\right\}$ [7], but here instead of just irrigation requirements we also model fertilization requirements, where each stage $s_{i} \in S$ is defined with a predetermined cumulative total soil water (TSW) and total soil fertilizer (TSF) range appropriate for Muvumba Valley. Water and fertilizer loss from each plot are monitored with a water level (WL) and fertilizer level (FL) sensors. Daily TSW/TSF calculations are summed to provide the cumulative value. As described in [1], the weather forecast is accessed by a Web 
service query and a calculated water need (CNW) and calculated fertilizer need (CNF) are obtained by accounting for the area of plot and fertilizer within soil. The system reacts to weather and soil variables to indicate a state of deficit, sufficient amount, or an excess of water or fertilizer according to the growth stage and thus define actions to take to satisfy the irrigation and fertilization demand. Target fertilization amounts by growth stage were taken from [9] and [10]. TSW/TWF is calculated each day following the method in [8] and summed as a way to track the targeted cumulative TSW/TWF for each stage.

$$
((T \text { SW/T SF })(s, a)) \leftarrow((T \text { SW/T SF })(S, a))+1
$$

Irrigation decisions are made at 2-day time steps and fertilization decisions are made at weekly time steps. A decision depends on the current stat $\mathrm{s} \in \mathrm{S}$, an action a $\in \mathrm{A}$, such as opening a valve, which changes the condition $\mathrm{C}$. The value called $\gamma$ is defined as when the water level and fertilizer level reaches the desired range between the threshold and the maximum. Whatever the decision, the new condition $\mathrm{C}(\mathrm{s}, \mathrm{a})$ is received and the state transits to $\mathrm{S}$. The cumulative T SW/TSF $\left(\mathrm{s}_{1}, \mathrm{a}_{1}\right)$ value of the state-action pair is updated with the calculated water and fertilizer needed (CNW/CNF). At state s, an action (a) is taken according to a greedy algorithm [6]. Then condition $\mathrm{C}$ is received and the state transits to $\mathrm{s}$. The TSW value of the stateaction pair $\left(\mathrm{s}_{1}, \mathrm{a}_{1}\right)$ is updated with the temporal difference to obtain CNW/CNF:

$$
C N W / C N F=C(s, a)+\gamma\left((T S W / T S F)\left(s_{1}, a_{1}\right)\right)-((T S W / T S F)(s, a))
$$

At each time step, all entries of the T SW/T SF water and fertilizer to the field are updated according to the Equation 1 and Equation 2 with a learning rate $\alpha$.

$$
((T S W / T S F)(s, a)) \leftarrow((T S W / T S F)(s, a))+\alpha((C N W / C N F)(s, a))
$$

These actions for generating TSW/TSF and taking control actions are outlined in Algorithm 1, where the program first translates time step i to 2 days for irrigation $[j, j+2]$ and 7 days for fertilization $[j, j+7]$. Then, it calculates irrigation water depth or fertilizer amount $\mathrm{I} j$ for day $\mathrm{j}$ according to current $\mathrm{TSW} / \mathrm{TSF}_{\mathrm{j}}\left(\mathrm{Total}_{\text {Soil }}\right.$ Water) and the CNW/CNF (calculated needed water and calculated needed fertilizer).

Algorithm 1 Algorithm for generating daily TSW/TSF record function

Generate daily TSW/TSF and irrigation and fertilization record function Cal((T SW=T SF )(i; a))

1. $\mathrm{j}=\left(\begin{array}{ll}\mathrm{i} & 1\end{array}\right) 2+1$ (irrigation)

2. $\mathrm{j}=\left(\begin{array}{ll}\mathrm{i} & 1\end{array}\right) 7+1$ (fertilization)

3. $\mathrm{Ij} \leftarrow$ Irrigation/Fertilization Amount (TSW/TSF, CNW/CNF, Weather)

4. if $I_{j}<$ Threshold then

5. $\mathrm{I}_{\mathrm{j}} \leftarrow 0$

6. end if

7. for $\mathrm{k}=\mathrm{j}+1 ; \mathrm{k}++$, while $\mathrm{k}<\mathrm{j}+3$ do $\mathrm{TWS}_{\mathrm{k}} \leftarrow$ CalT SW $\left(\mathrm{TSW}_{(\mathrm{k} 1)} ; \mathrm{I}_{\mathrm{k}}{ }_{1} ; \mathrm{ET}_{\mathrm{k}}{ }_{1} ; \mathrm{C}_{\mathrm{k}}{ }_{1}\right)$ do irrigation

8. end for

9. for $\mathrm{k}=\mathrm{j}+1 ; \mathrm{k}++$, while $\mathrm{k}<\mathrm{j}+14$ do $\left.\mathrm{T} \mathrm{SF}_{\mathrm{k}} \leftarrow \mathrm{CalT} \mathrm{SF}\left(\mathrm{T} \mathrm{SF}_{(\mathrm{k}}{ }_{1}\right) ; \mathrm{I}_{\mathrm{k}} \quad 1 ; \mathrm{ET}_{\mathrm{k}} \quad{ }_{1} ; \mathrm{C}_{\mathrm{k}}{ }_{1}\right)$ do fertilization

\section{0. end for}

11. Return ((T SW=T SF $\left.)_{j+2}\right)$ and $\mathrm{I}_{\mathrm{j}}$

When there is a delay of irrigation or fertilization because of technical problem, we will use the temporal difference-learning algorithm SARSA $(\lambda)$ [5] instead of the MCP. This policy is a state-action pair tuple which maps the action to be taken at each state and control method chooses the action for each state during learning by following MDP rules applied at every time step and by letting the agent transition from one stateaction pair to another. The product of $\gamma$ and $\lambda$ discounts the field so that conditions is reached at later time steps are updated according to the relevance of previous state-action pair.

$$
((\mathrm{T} \mathrm{SW} / \mathrm{T} \mathrm{SF})(\mathrm{s}, \mathrm{a})) \leftarrow \gamma \lambda((\mathrm{T} \mathrm{SW} / \mathrm{T} \mathrm{SF})(\mathrm{s}, \mathrm{a}))
$$

When SARSA is running, the time step is set to be 2 days. SARSA requires a priori the water demand and nutrient demand for each stage of the rice crop because each stage has its own minimum and maximum levels for optimal crop growth. Algorithm 2 provides a complete description of the learning processes, which indicates the operation with MCP when the system is fully functional and operation with SARSA when there is a system fault. The program starts with a randomly generated T SW/T SF table. At each time step, the 
agent takes an action and runs Equation 2 to obtain CNW/CNF. T SW/T SF and updating the irrigation or fertilization record for the current time step uses Equation 3 or 4 . The model can be adapted to other crops by adjusting IoT sensor types and model parameters.

Algorithm 2 Markov and SARSA ( ) in Irrigation

1: Initialize ((T SW/T SF)(s, a)) randomly

2: for All $((\mathrm{CNW} / \mathrm{CNF})(\mathrm{s}, \mathrm{a}))$ do

3: $((\mathrm{T} \mathrm{SW} / \mathrm{T} \mathrm{SF})(\mathrm{s}, \mathrm{a}) \leftarrow 0$

4: for $\mathrm{i}=1, \mathrm{i}++$ while $\mathrm{i}<\mathrm{n}+1$ do $\mathrm{s} \leftarrow$ State $(\mathrm{i},((\mathrm{T} \mathrm{SW} / \mathrm{T}$ SF $) \mathrm{i}))$ do

5: Take action a

6: [(T SW/T SF $) i+1$, Ii $] \leftarrow((C N W / C N F)(i, a)) S_{1} \leftarrow$ State(i,((T SW/T SF $\left.\left.) i\right)\right)$

7: $\mathrm{a}_{1} \leftarrow$ greedy $((\mathrm{T} \mathrm{SW} / \mathrm{T} \mathrm{SF}), \mathrm{Si}+1)$

8: $(\mathrm{CNW} / \mathrm{CNF}) \leftarrow \mathrm{C}(\mathrm{s}, \mathrm{a})+\gamma\left((\mathrm{T} \mathrm{SW} / \mathrm{T} \mathrm{SF})\left(\mathrm{s}_{1}, \mathrm{a}_{1}\right)\right)-((\mathrm{T} \mathrm{SW} / \mathrm{T} \mathrm{SF})(\mathrm{s}, \mathrm{a}))$

9: $((\mathrm{T} \mathrm{SW} / \mathrm{T} \mathrm{SF})(\mathrm{s}, \mathrm{a})) \leftarrow((\mathrm{T} \mathrm{SW} / \mathrm{T} \mathrm{SF})(\mathrm{s}, \mathrm{a}))+1$

10: for All s\&a do

11: $(($ T SW/T SF $)(s, a)) \leftarrow(($ T SW/T SF $)(s, a))+((C N W / C N F)(s, a))$

12: $((\mathrm{T} \mathrm{SW} / \mathrm{T} \mathrm{SF})(\mathrm{s}, \mathrm{a})) \leftarrow \gamma \lambda((\mathrm{T} \mathrm{SW} / \mathrm{T} \mathrm{SF})(\mathrm{s}, \mathrm{a}))$

13: end for

14: end for

15: end for

16: Until ((T SW/T SF)(s, a)) policy sufficiently stabilized

\section{Results}

To evaluate the effectiveness of the decision modeling system, we used historical data from Meteo Rwanda for rainfall, temperature, and evaporation from March 2019 to January 2020. These data are from the Nyagatare weather station in Eastern province of Rwanda adjacent to the Muvumba Rice Plantation.

The Nyagatare weather station data provide data relevant to irrigation and fertilization decisions. The soil type and $\mathrm{pH}$ value are taken from [11]. Figure 2 shows the system results for the operational system of calculated needed fertilizer (NPK, Urea) and needed water level for every day as it was formulated in Matlab. The system will communicate the irrigation and fertilization status to the farmer to communicate what is happening in his/her rice field. The findings of the system simulation indicates an average of $31.62 \mathrm{~kg} / \mathrm{ha}$ of NPK and $21.4 \mathrm{~kg} / \mathrm{ha}$ of urea needed for each stage. These fertilization amounts are less than the government allotments of fertilizers the farmers receive. This may indicate over fertilization to be occurring at Muvumba Valley rice plantation.

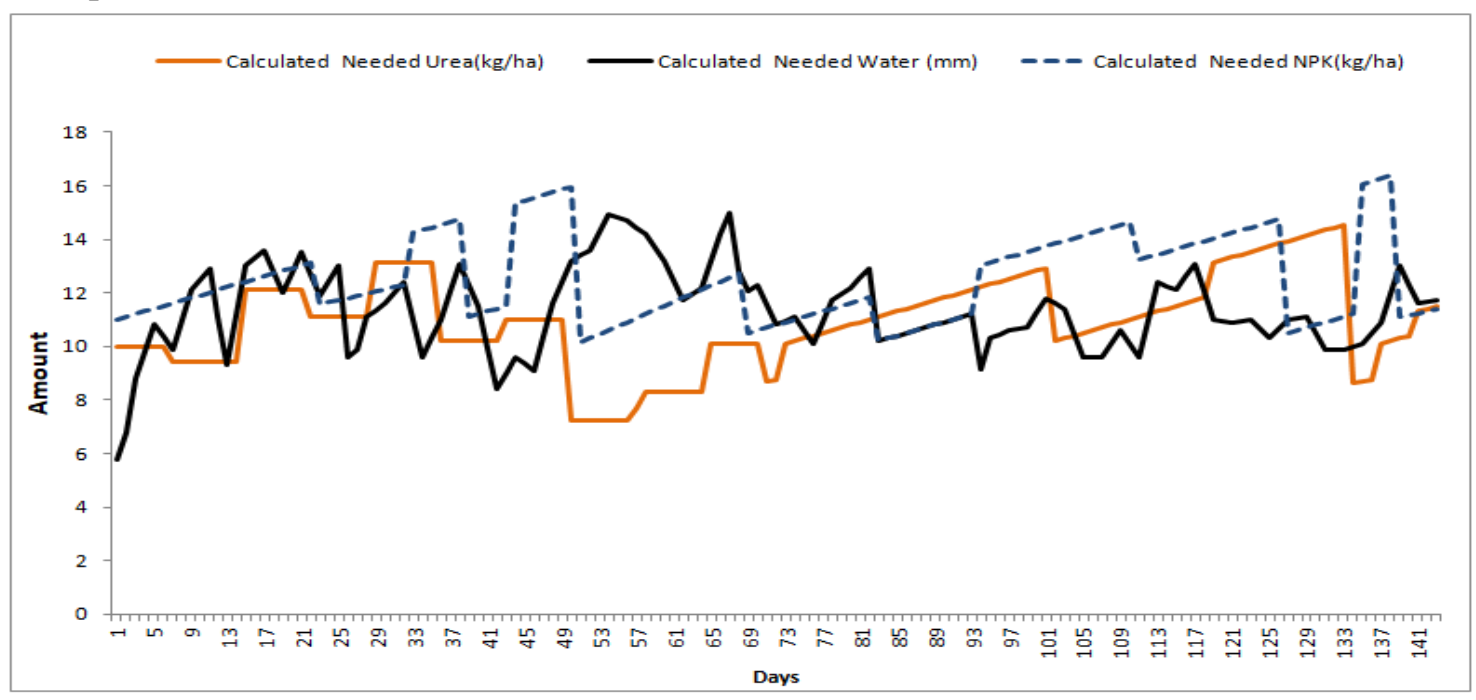

Fig. 2: Result for Matlab simulation of one season using MCP.

The simulation of a fault is done for the same set of data used for the MCP simulation, but assuming a fault in the system for the whole season without any sensor updates. This worst-case scenario for the SARSA 
model thus relies on prior knowledge of water depth and fertilizer requirements for the growth stages, typical soil nutrient content, and climatological temperature and rainfall. Figure 3 shows water and fertilization amounts recommended to a single example plot. The irrigation amounts by stage generally follow needs by growth stage. Overall, the fertilizer recommendations ranged from $91.24 \mathrm{~kg} / \mathrm{ha}$ to $113.7 \mathrm{~kg} / \mathrm{ha}$ for NPK for the whole season. For urea the recommendations ranged from $69.34 \mathrm{~kg} / \mathrm{ha}$ to $86.67 \mathrm{~kg} / \mathrm{ha}$ for whole season. Similar to the MCP results, for both fertilizer types the recommendations are less than the allotments farmers now put on their fields.

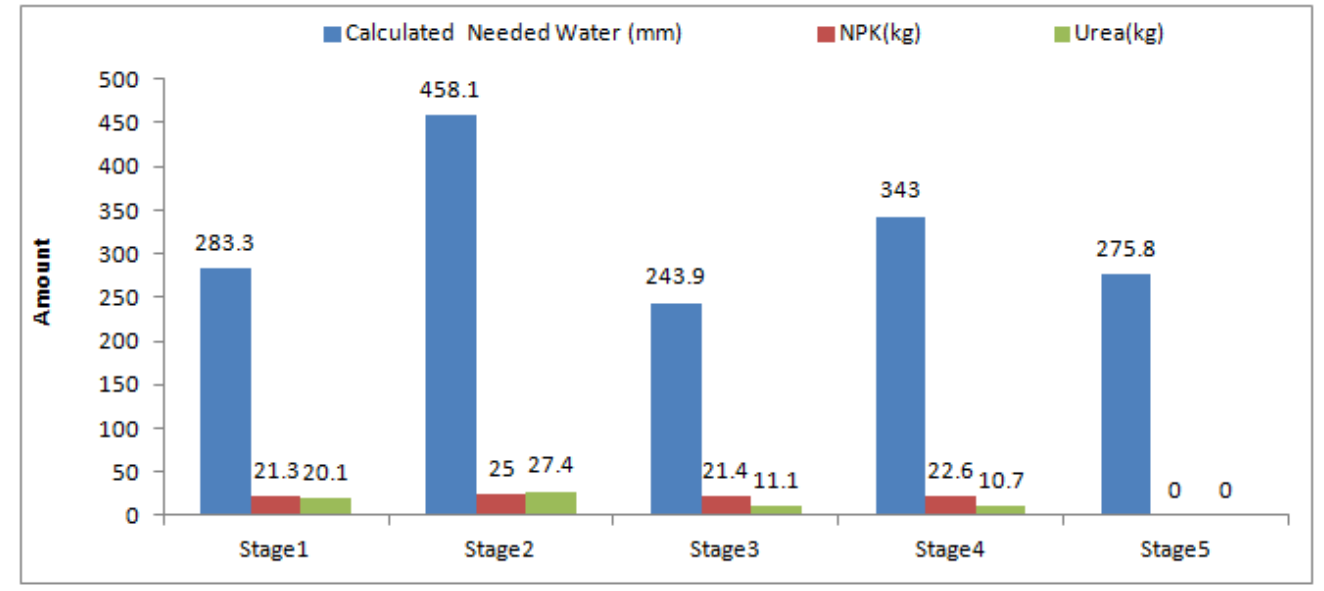

Fig. 3: Results using SARSA for the five rice growth stages. The method communicates reasonable irrigation and fertilization amounts to the farmer for manual action.

\section{Discussion and Future Work}

We have described adapting MCP and SARSA modeling to improve irrigation and fertilization actions to increase Rwandan rice production using an IoT approach. The simulation results suggest reasonable irrigation and fertilization decisions are made by the system and these decisions appear to be more efficient than the current irrigation and fertilization practices at Muvumba Valley. Farmers can remotely interact with irrigation and fertilization controls based on their own expert knowledge combined with the system data. Fault tolerance improves resiliency by switching modeling modes. The IoT system has promise to minimize the human effort, reduce water and fertilizer use, improve productivity, and increased return on investment. The data collected over time are stored for analysis to improve decision making over time. The system can also work for the Alternate Wetting and Drying irrigation method by accounting for wet and dry periods in the growth stages. Future work can include integrating remote sensing data from drones or satellites and economic data to seek to maximum benefits at low cost.

\section{References}

[1]. Peace Bamurigire, Anthony Vodacek, Andras Valko, and Said Rutabayiro Ngoga. Simulation of Internet of Things Water Management for Efficient Rice Irrigation in Rwanda. Agriculture 2020, 10(10), 431 , https://doi.org/10.3390/agriculture10100431.

[2]. Institute of Statistics of Rwanda. http://www.statistics.gov.rw/publication/seasonal-agricultural-survey-2019annual-report, retrieved 18 June 2020.

[3]. Pascal RushemukaL. BockJ.G. Mowo. (2014).Land Use Change Impacts on Water Erosionin Rwanda.Sustainability2020,12, 50; doi:10.3390/su12010050, www.mdpi.com/journal/sustainability.

[4]. Matis, J.H., T. Saito, W. E. Grant. (1985). A Markov Chain Approach to Crop Yield Forecasting. Agricultural Systems. 18, 171-187.

[5]. Sutton, R.S., and A.G. Barto. (1998). Reinforcement Learning. An Introduction. MIT Press, 1st Ed. The MIT Press. Cambridge, MA.

[6]. Bednorz, W. (2008). Greedy Algorithms. IntechOpen. doi: 10.5772/92

[7]. Alvarez-Herrera, J.G., L.P. Pinzon-Gomez, and J.E. Velez. (2017). Growth and production of rice (Oryza Sativa 
L.) under different fertilization plans with silicon. Ingenieria e Investigacion. 37, 7-15, doi:10.15446/ing.investig.v37n1.59344.

[8]. Resources, M. o. (2013). Agriculture Mechanization Strategy for Rwanda. Kigali: Irrigation and Mechanization Task Force.

[9]. Ahmed, N., D. De, and I. Hussain. (2018). Internet of Things (IoT) for Smart Precision Agriculture and Farming in Rural Areas. IEEE Internet of Things Journal 5, 4890-4899. doi:10.1109/JIOT.2018.2879579.

[10]. Bijay-Singh and V.K. Singh.(2017). Fertilizer Management in Rice. Springer International Publishing AG.B.S. Chauhan et al. (eds.), Rice Production Worldwide, DOI 10.1007/978-3-319-47516-5-10.

[11]. Niyonkuru, R, R. Sankaranarayanan, S.K. Pande, D. Das. 2019. Application of FAO-CROPWAT software for modeling irrigation schedule of rice in Rwanda. Rwanda Journal of Agricultural Sciences. 1(1):7-13. 